PROCEEDINGS OF THE

AMERICAN MATHEMATICAL SOCIETY

Volume 135, Number 4, April 2007, Pages 1181-1186

S 0002-9939(06)08661-8

Article electronically published on October 18, 2006

\title{
HYPERBOLIC CONVEXITY AND THE ANALYTIC FIXED POINT FUNCTION
}

\author{
ALEXANDER YU. SOLYNIN
}

(Communicated by Juha M. Heinonen)

\begin{abstract}
We answer a question raised by D. Mejía and Ch. Pommerenke by showing that the analytic fixed point function is hyperbolically convex in the unit disc.
\end{abstract}

Let $\mathbb{D}$ be the unit disc in $\mathbb{C}$ and let $\varphi: \mathbb{D} \rightarrow \mathbb{D}$ be an analytic map. The goal of this note is to prove the following.

Theorem 1. If $\varphi(0) \neq 0$, then the set $F=\{z:|z|<|\varphi(z)|\}$ is a hyperbolically convex domain in $\mathbb{D}$. It is hyperbolically strictly convex unless $\varphi$ is a Möbius map, in which case $F$ is a hyperbolic half-plane.

Thus this theorem solves Problem 1 posed by D. Mejía and Ch. Pommerenke in [7, where they initiated the study of an analytic fixed point function of $\varphi$ and its applications to Probability Theory; see [7, 8]. For the case when the function $g(z)=z / \varphi(z)$ is univalent in $\mathbb{D}$, this theorem was proved in [7. The fixed point function of $\varphi$ is defined in [7] as the solution to the functional equation

$$
w \varphi(f(w))=f(w) \quad \text { for } w \in \mathbb{D} .
$$

Therefore $f(w)$ is the inverse function of $w=z / \varphi(z)$. It was shown in [7] that $f$ maps $\mathbb{D}$ conformally and one-to-one onto the domain $F$ defined in Theorem 1 . which in its turn is starlike with respect to $z=0$.

Before proving Theorem 1 we remind the reader of some basic facts of hyperbolic geometry in the unit disc $\mathbb{D}$ supplied with the hyperbolic metric, $\rho$, defined by

$$
d \rho(z)=\frac{|d z|}{1-|z|^{2}}, \quad z \in \mathbb{D} .
$$

As is well known the hyperbolic geodesic intervals $\left(z_{1}, z_{2}\right)$ from $z_{1}$ to $z_{2}$ are open arcs of circles, which are orthogonal to the unit circle $\mathbb{T}=\partial \mathbb{D}$. A domain $D \subset \mathbb{D}$ is called hyperbolically convex if $\left(z_{1}, z_{2}\right) \subset D$, whenever $z_{1}, z_{2} \in D$. It is hyperbolically strictly convex if $\left(z_{1}, z_{2}\right) \subset D$, whenever $z_{1}, z_{2} \in \partial D$.

Received by the editors November 17, 2005.

2000 Mathematics Subject Classification. Primary 30C55, 30F45.

Key words and phrases. Analytic fixed point function, hyperbolic convexity, Riemann surface, hyperbolic metric.

This research was supported in part by NSF grant DMS-0412908.

(C)2006 American Mathematical Society Reverts to public domain 28 years from publication 
Finally, we want to mention that Theorem 1 complements the classical PickSchwarz lemma, which says that $\varphi$ is a contraction in the hyperbolic metric, i.e.,

$$
\left|\frac{\varphi\left(z_{1}\right)-\varphi\left(z_{2}\right)}{1-\varphi\left(z_{1}\right) \overline{\varphi\left(z_{2}\right)}}\right| \leq\left|\frac{z_{1}-z_{2}}{1-z_{1} \bar{z}_{2}}\right|
$$

for all $z_{1}, z_{2} \in \mathbb{D}$ with the sign of equality only for Möbius self maps of $\mathbb{D}$.

Proof of Theorem 1. Let $g(z)=z / \varphi(z)$. Then $g$ is a meromorphic function in $\mathbb{D}$ whose only zero is at $z=0$. Let $\mathcal{R}$ be the Riemann surface of $g^{-1}$. Then $\mathcal{R}$ is a hyperbolic simply connected Riemann surface over $\overline{\mathbb{C}}$ possibly with branch points. Since $\liminf |z| \rightarrow 1|g(z)| \geq 1$ and $g(z) \neq 0$ when $z \neq 0$, it follows that $\mathcal{R}$ has exactly one sheet, called $\mathcal{R}^{0}$, over $\mathbb{D}$. Then the fixed point function $f$ maps $\mathcal{R}^{0}$ onto $F$.

Composing $g$ with the Möbius map $\psi(w)=i \frac{w-1}{w+1}$ we transplant the problem into the $\zeta$-plane. Then $\zeta=\psi(g(z))$ maps $\mathbb{D}$ onto the hyperbolic Riemann surface $\mathcal{R}_{\zeta}$ over $\overline{\mathbb{C}}_{\zeta}$, which has exactly one sheet $\mathcal{R}_{\zeta}^{0}$ over the lower half-plane $\mathbb{H}_{-}=\{\zeta: \Im \zeta<$ $0\}$.

It is important to emphasize here that $F$ is hyperbolically convex/strictly convex with respect to $\mathbb{D}$ if and only if $\mathcal{R}_{\zeta}^{0}$ is hyperbolically convex/strictly convex with respect to $\mathcal{R}_{\zeta}$. Now the desired conclusion about hyperbolic convexity follows from Lemma 2 below.

By the same lemma, $\mathcal{R}_{\zeta}^{0}$ (and therefore $F$ ) is hyperbolically strictly convex unless the composition $\psi \circ g$ maps $\mathbb{D}$ conformally onto the complex sphere $\overline{\mathbb{C}}$ slit along a closed segment of the extended real axis $\overline{\mathbb{R}}$. Then an easy calculation shows that $\varphi$ must be a Möbius self map of $\mathbb{D}$, in which case $F$ is a hyperbolic half-plane; see [7, Example 3.1].

As we mentioned above, D. Mejía and Ch. Pommerenke [7] have proved Theorem 1 for the special case that the function $g(z)=z / \varphi(z)$ is univalent. To prove this case, they employed V. Jørgensen's [6] convexity theorem for circular discs embedded in a planar multiply connected domain. The following lemma, its corollary, and Lemma 2, needed to complete the proof of Theorem 1, extend Jørgensen's results for the case of simply connected Riemann surfaces. In our proofs we use extensively basic results about the hyperbolic metric on Riemann surfaces. Necessary properties can be found, for example, in 2, 4, Chapter 2], and [5, Chapter 9]. The details of our proofs are similar to those in [6] and [10].

Lemma 1. Let $\mathcal{R}$ be a hyperbolic simply connected Riemann surface over $\overline{\mathbb{C}}$, which has exactly one sheet $\mathcal{R}^{0}$ over the lower half-plane $\mathbb{H}_{-}$. Let $\rho\left(P_{z}\right)|d z|$ denote the hyperbolic metric on $\mathcal{R}$ at the point $P_{z}$ above the point $z$ in the plane.

If $\Im z>0$, then

$$
\rho\left(P_{\bar{z}}\right) \leq \rho\left(P_{z}\right)
$$

for $P_{\bar{z}} \in \mathcal{R}^{0}$ and for every point $P_{z}$ on $\mathcal{R}$ lying above $z$.

In addition, if $P_{z} \in \mathcal{R}$ and $z$ is real, then

$$
\frac{\partial \rho\left(P_{z}\right)}{\partial y} \geq 0, \quad \text { where } z=x+i y .
$$

If equality occurs in (11) or (2), then $\mathcal{R}$ coincides with the complex sphere $\overline{\mathbb{C}}$ slit along a closed segment of $\overline{\mathbb{R}}$. 
Proof. Let $g: \mathbb{D} \rightarrow \mathcal{R}$ be a meromorphic function generating $\mathcal{R}$. For $0<r<1$, let $\mathcal{R}_{r}$ denote the Riemann surface over $g(|\zeta|<r)$ and let $\rho_{r}$ be the hyperbolic metric of $\mathcal{R}_{r}$. Since $\mathcal{R}_{r}$ is a proper subset of $\mathcal{R}, \rho\left(P_{z}\right)<\rho_{r}\left(P_{z}\right)$ for every point $P_{z} \in \mathcal{R}_{r}$, where $\rho$ is finite. In addition, $\rho_{r}\left(P_{z}\right) \rightarrow \rho\left(P_{z}\right)$ as $r \rightarrow 1^{-}$.

We will employ the well-known fact that the functions $u=\log \rho$ and $u_{r}=\log \rho_{r}$ satisfy the following Liouville's equation:

$$
\Delta u\left(P_{z}\right)=4 e^{2 u\left(P_{z}\right)}, \quad P_{z} \in \mathcal{R}, \quad \Delta u_{r}\left(P_{z}\right)=4 e^{2 u_{r}\left(P_{z}\right)}, \quad P_{z} \in \mathcal{R}_{r},
$$

where $\Delta$ stands for the Laplacian with respect to $z=x+i y$.

Now we proceed by contradiction. Assume that $\rho\left(P_{\bar{z}_{0}}\right)>\rho\left(P_{z_{0}}\right)$ for some point $P_{z_{0}} \in \mathcal{R}$ with $\Im z_{0}>0$. Since $\rho_{r}\left(P_{z_{0}}\right) \rightarrow \rho\left(P_{z_{0}}\right)$ as $r \rightarrow 1^{-}$, we can choose $0<r_{0}<1$ such that $\rho\left(P_{\bar{z}_{0}}\right)>\rho_{r_{0}}\left(P_{z_{0}}\right)$. In addition, we may assume without loss of generality that $g^{\prime}(\zeta) \neq 0$ on $C_{0}=\left\{\zeta:|\zeta|=r_{0}\right\}$. Then $L_{0}=\partial \mathcal{R}_{r_{0}}$ is a closed, in general non-Jordan, analytic curve over $\overline{\mathbb{H}}_{+}=\{z: \Im z \geq 0\}$. Therefore $u\left(P_{z}\right)$ is continuous on $C_{0}$ and $u_{r_{0}}\left(P_{z}\right) \rightarrow+\infty$ as $P_{z} \rightarrow L_{0}$.

Since $\mathcal{R}$ covers the lower half-plane precisely once, for every point $P_{z} \in \mathcal{R}$ with $\Im z>0$, there exists precisely one point $P_{\bar{z}}$ in $\mathcal{R}$. Hence $u\left(P_{\bar{z}}\right)$ is a well-defined, finite, and continuous function of $z$, when $z$ varies such that $P_{z}$ is in $\mathcal{R}_{r_{0}}^{+}:=\left\{P_{z} \in\right.$ $\left.\mathcal{R}_{r_{0}}: \Im z>0\right\}$. It is also important to note that $\mathcal{R}$ has no branch points over the lower half-plane and, if $B$ is a branch point of $\mathcal{R}_{r_{0}}$, then $u_{r_{0}}\left(P_{z}\right) \rightarrow+\infty$ as $P_{z} \rightarrow B$.

Let

$$
v\left(P_{z}\right)=u\left(P_{\bar{z}}\right)-u_{r_{0}}\left(P_{z}\right), \quad P_{z} \in \mathcal{R}_{r_{0}}^{+} .
$$

Then by our assumption,

$$
m=\sup v\left(P_{z}\right)>0
$$

where the supremum is taken over all $P_{z} \in \mathcal{R}_{r_{0}}^{+}$. Let $E=\left\{P \in \mathcal{R}_{r_{0}}^{+}: v(P)=m\right\}$. Note that $v\left(P_{z}\right) \rightarrow-\infty$ as $P_{z} \rightarrow L_{0}$ or $P_{z} \rightarrow B$, where $B$ is a branch point of $\mathcal{R}_{r_{0}}$. Also $v\left(P_{z}\right)=u\left(P_{z}\right)-u_{r_{0}}\left(P_{z}\right)<0$ for $P_{z} \in \mathcal{R}_{r_{0}}$ and $\Im z=0$ because the closure of $\mathcal{R}_{r_{0}}$ is contained in $\mathcal{R}$. Therefore $v$ achieves its maximum in (5) at some regular inner point $P_{z_{1}}$ of $\mathcal{R}_{r_{0}}^{+}$.

Thus, $E \neq \emptyset$. Since $v$ is continuous in $\mathcal{R}_{r_{0}}^{+}$except at its branch points, it follows that $E$ is closed in $\mathcal{R}_{r_{0}}^{+}$. We claim that $E$ is also open in $\mathcal{R}_{r_{0}}^{+}$. Indeed, let $P_{z_{1}} \in E$. Since $v$ is continuous at $P_{z_{1}}$ and $v\left(P_{z_{1}}\right)>0$, there is a flat disc $U \subset \mathcal{R}_{r_{0}}^{+}$centered at $P_{z_{1}}$ such that $v>0$ on $U$. From (3) -(5), we get

$$
\Delta v\left(P_{z}\right)=4\left(e^{2 u\left(P_{\bar{z}}\right)}-e^{2 u_{r_{0}}\left(P_{z}\right)}\right)>0 \text { for any } P_{z} \in U \text {. }
$$

Therefore, $v$ is subharmonic on $U$. Since $v$ takes its maximal value at the center of the disc $U$, the strong maximum principle implies that $v \equiv m$ on $U$.

Now since $E \neq \emptyset$ is closed and open in $\mathcal{R}_{r_{0}}^{+}$, it follows that $E$ coincides with some connected component of $\mathcal{R}_{r_{0}}^{+}$. Since every such component has a common boundary interval with $\mathcal{R}_{0}$, the latter contradicts the condition that $v\left(P_{z}\right)<0$ for all $P_{z} \in \mathcal{R}_{r_{0}}$ such that $\Im z=0$.

Since the assumption that $\rho\left(P_{\bar{z}_{0}}\right)>\rho\left(P_{z_{0}}\right)$ for some $P_{z_{0}} \in \mathcal{R}$ for $\Im z_{0}>0$ leads to a contradiction, inequality (11) follows. This also implies (2) after an easy differentiation.

Now that the non-strict inequalities are established, the assertion on the equality cases can be proved along the same lines. Let $v=u\left(P_{\bar{z}}\right)-u\left(P_{z}\right)$, where $u$ is defined above. Assuming that $v\left(P_{z_{0}}\right)=0$ for some $P_{z_{0}} \in \mathcal{R}^{+}$, where $\mathcal{R}^{+}=\left\{P_{z} \in \mathcal{R}\right.$ : 
$\Im z>0\}$, we consider the non-empty set $E=\left\{P \in \mathcal{R}^{+}: v(P)=0\right\}$. Since $v$ is continuous everywhere in $\mathcal{R}$ except at its branch points, the set $E$ is closed with respect to $\mathcal{R}^{+}$. To prove that $E$ is open, we choose $P_{z_{0}} \in E$. Since $\rho\left(P_{\bar{z}_{0}}\right)$ is finite, so is $\rho\left(P_{z_{0}}\right)$. Hence $P_{z_{0}}$ is not a branch point of $\mathcal{R}$. Therefore there is a flat disc $U$ embedded in $\mathcal{R}$ with the center at $P_{z_{0}}$ such that $v\left(P_{z}\right) \leq 0$ everywhere on $\bar{U}$. By (3), we have

$$
\Delta v\left(P_{z}\right)=4\left(e^{2 u\left(P_{\bar{z}}\right)}-e^{2 u\left(P_{z}\right)}\right) .
$$

Applying the standard mean value theorem to the right-hand side of this equation, we obtain

$$
\Delta v\left(P_{z}\right)+c\left(P_{z}\right) v\left(P_{z}\right)=0
$$

with some function $c\left(P_{z}\right) \leq 0$ bounded on $\bar{U}$. It is known that non-positive solutions of (6) satisfy the strong maximum principle; see [12, Theorem 2.6]. Since $v\left(P_{z_{0}}\right)=0$ the latter implies that $v \equiv 0$ on $\bar{U}$.

Thus, $E \neq \emptyset$ is open and closed in $\mathcal{R}^{+}$. Therefore $E$ coincides with some connected component, say $\widetilde{\mathcal{R}}^{+}$, of $\mathcal{R}^{+}$and $\rho\left(P_{\bar{z}}\right)=\rho\left(P_{z}\right)$ for all $P_{z} \in \widetilde{\mathcal{R}}^{+}$. In particular, $\widetilde{\mathcal{R}}$ does not have branch points and does not have boundary points over the upper half-plane. Since $\mathcal{R}$ is hyperbolic and simply connected, the latter easily implies that $\widetilde{\mathcal{R}}^{+}$is a non-ramified cover over the upper half-plane, which is attached to $\mathcal{R}_{0}$ along some open interval, say $I$, of the extended real axis. Let $P_{z} \in \widetilde{\mathcal{R}}^{+}$with $z=x+i y, x \in \overline{\mathbb{R}} \backslash I$. Then $\rho\left(P_{\bar{z}}\right)=\rho\left(P_{z}\right) \rightarrow+\infty$ as $y \rightarrow 0^{+}$. This implies that except for $\widetilde{\mathcal{R}}^{+}$there are no other connected components of $\mathcal{R}^{+}$. Therefore if $\rho\left(P_{\bar{z}_{0}}\right)=\rho\left(P_{z_{0}}\right)$ for some $P_{z_{0}} \in \mathcal{R}^{+}$, then $\mathcal{R}$ must coincide with the complex sphere $\overline{\mathbb{C}}$ slit along a closed segment of the extended real line.

Now assume that $\frac{\partial \rho\left(P_{z_{0}}\right)}{\partial y}=0$ for some $P_{z_{0}} \in \mathcal{R}$ with $z_{0}=x_{0} \in \mathbb{R}$. Then $P_{z_{0}}$ is not a branch point of $\mathcal{R}$, and therefore there is a flat disc $U$ over $\left\{z:\left|z-\left(x_{0}+i \varepsilon\right)\right|<\right.$ $\varepsilon\}$ with some $\varepsilon>0$ embedded in $\mathcal{R}$ such that $P_{z_{0}}$ is a boundary point of $U$. As we have seen above, $v=u\left(P_{\bar{z}}\right)-u\left(P_{z}\right)$ is a non-negative solution to (6) in $U$. Since

$$
\frac{\partial v\left(P_{z_{0}}\right)}{\partial y}=-2 \rho^{-1}\left(P_{z_{0}}\right) \frac{\partial \rho\left(P_{z_{0}}\right)}{\partial y}=0,
$$

Hopf's lemma for (6), (see [12, Theorem 2.6]) implies that $v \equiv 0$ in $U$. Therefore, $\rho\left(P_{\bar{z}}\right)=\rho\left(P_{z}\right)$ for some $P_{z} \in \mathcal{R}$ such that $\Im z>0$, which implies the desired conclusion.

The Riemann surface $\mathcal{R}$ defined in Lemma 1 has exactly one sheet over every half-plane $H_{c}^{-}:=\{z: \Im z<c\}$ with $c<0$. This and Lemma 1 yield the following result.

Corollary 1. If $\Im z_{0}<0$, then

$$
\frac{\partial \rho\left(P_{z_{0}}\right)}{\partial y}>0
$$

If in addition, $\alpha>0$ and $P_{z_{0}+i \alpha} \in \mathcal{R}$, then

$$
\rho\left(P_{z_{0}-i \alpha}\right)<\rho\left(P_{z_{0}+i \alpha}\right) .
$$

Lemma 2. Under the assumptions of Lemma 1, the domain $\mathcal{R}^{0}$ is hyperbolically convex with respect to $\mathcal{R}$. It is hyperbolically strictly convex unless $\mathcal{R}$ coincides with the complex sphere $\overline{\mathbb{C}}$ slit along a closed segment of $\overline{\mathbb{R}}$. 
For every $\alpha>0$, the domain $\mathcal{R}_{\alpha}=\left\{P_{z} \in \mathcal{R}: \Im z<-\alpha\right\}$ is hyperbolically strictly convex with respect to $\mathcal{R}$.

Proof. Let $P_{z_{1}}$ and $P_{z_{2}}$ be distinct points in $\mathcal{R}^{0}$, where $z_{1}=x_{1}+i y_{1}, z_{2}=x_{2}+i y_{2}$ and $y_{1} \geq y_{2}$. Let $\gamma$ be the geodesic arc on $\mathcal{R}$ joining $P_{z_{1}}$ and $P_{z_{2}}$. Let $\gamma^{+}$and $\gamma^{-}$denote the projections of $\gamma$ onto the half-planes $H_{y_{1}}^{+}=\left\{z: \Im z>y_{1}\right\}$ and $\bar{H}_{y_{1}}^{-}=\left\{z: \Im z \leq y_{1}\right\}$, respectively. Let $\tilde{\gamma}^{+}$be the reflection of $\gamma^{+}$with respect to the horizontal line $\left\{z: \Im z=y_{1}\right\}$. Then $\gamma^{-}$and $\tilde{\gamma}^{+}$can be glued together to obtain a piecewise smooth, in general non-Jordan, curve $\tilde{\gamma}$ on $\bar{H}_{y_{1}}^{-}$joining $P_{z_{1}}$ and $P_{z_{2}}$. If $\gamma^{+} \neq \emptyset$, then using (7) we obtain

$$
\text { hyp.length } \tilde{\gamma}=\int_{\tilde{\gamma}} \rho\left(P_{z}\right)|d z|<\int_{\gamma} \rho\left(P_{z}\right)|d z|=\text { hyp.length } \gamma,
$$

which is impossible since $\gamma$ is a geodesic by assumption. Hence, $\gamma^{+}=\emptyset$ and therefore $\mathcal{R}^{0}$ is hyperbolically convex with respect to $\mathcal{R}$.

Now we assume that $z_{1}$ and $z_{2}$ are distinct points on $\overline{\mathbb{R}}$. The lifts $P_{z_{1}}$ and $P_{z_{2}}$ on $\mathcal{R}$ will be considered as boundary points of $\mathcal{R}^{0}$. Here we admit the case when one or both of these points belong to the boundary of $\mathcal{R}$. Let $\gamma$ be the geodesic interval, possibly of infinite hyperbolic length, joining $P_{z_{1}}$ and $P_{z_{2}}$ on $\mathcal{R}$. It follows from our previous arguments that $\gamma$ lies over the closed lower half-plane, and if two points of $\gamma$ belong to $\mathcal{R}^{0}$, then the whole arc of $\gamma$ joining these points belongs to $\mathcal{R}^{0}$. This easily implies that $\gamma$ itself belongs to $\mathcal{R}^{0}$ unless $\gamma$ is a proper interval of the extended real axis. In the latter case let $g: \mathbb{D} \rightarrow \mathcal{R}$ be a meromorphic function generating $\mathcal{R}$. Since $\gamma$ is a geodesic interval on $\mathcal{R}, g^{-1}(\gamma)$ is a geodesic interval on $\mathbb{D}$ and therefore $g^{-1}(\gamma)$ is an arc of a circle orthogonal to $\mathbb{T}$. Now the Schwarz symmetry principle implies that $\mathcal{R}$ coincides with the sphere $\overline{\mathbb{C}}$ slit along a closed segment of $\overline{\mathbb{R}}$.

The last statement of the lemma obviously follows from its first part.

As it was noted in [7, Theorem 1 yields some important consequences. First we mention that length $\partial F \leq \pi^{2}$, by B. Brown Flinn's isoperimetric inequality for the perimeter of hyperbolically convex sets on $\overline{\mathbb{D}}$; see 1 . In particular, $f^{\prime} \in H^{1}$. This implies (see discussion in [7, Section 6]) that $a_{n, n} \rightarrow 0$, where $a_{n, n}$ are Taylor coefficients of $w f^{\prime}(w) / f(w)$ and $f(w)$ is the fixed point function as defined above.

Remarks. (1) Let $D$ be a domain on a simply connected Riemann surface $\mathcal{R}$ over $\overline{\mathbb{C}}$, let $D^{+}=\left\{P_{z} \in \mathcal{R}: \Im z>0\right\}, D^{-}=\left\{P_{z} \in \mathcal{R}: \Im z<0\right\}$, and let $D_{z}^{+}$and $D_{z}^{-}$denote the projections of $D^{+}$and $D^{-}$onto the upper and lower half-planes of $\mathbb{C}$, respectively. Assume that $D^{-}$is a single sheeted cover over $D_{z}^{-}$and that $D_{z}^{+*}:=\left\{z: \bar{z} \in D_{z}^{+}\right\} \subset D_{z}^{-}$. Then the assertion of Lemma 1 and the first part of Lemma 2 still remain true for the hyperbolic metric of $D$.

(2) The condition $D_{z}^{+*} \subset D_{z}^{-}$says, in the language of the theory of symmetrization, that $D$ is a polarized domain, which means that a larger portion of $D$ is concentrated in the lower half-plane.

If the original domain $D$ on a Riemann surface $\mathcal{R}$ is not polarized, there is a procedure called polarization, which transforms $D$ into a polarized domain on $\mathcal{R}$, in such a way that the hyperbolic metric, lowest eigenvalue, and some other characteristics of $D$ change monotonically under this transformation. We refer to [3], 9], 10] for further details. 
There is a huge literature about symmetrization and polarization in $\mathbb{R}^{n}$, but very little is written about these transformations in the context of Riemann surfaces; see [9], 11. So this message is to notify interested readers that an appropriate technique is available.

\section{ACKNOWLedGement}

The author wishes to thank Professor R. W. Barnard (Texas Tech University) for bringing this problem and the papers of D. Mejía and Ch. Pommerenke to his attention.

\section{REFERENCES}

1. B. Brown Flinn, Hyperbolic convexity and level sets of analytic functions. Indiana Univ. Math. J. 32 (1983), 831-841. MR0721566 (85b:30010)

2. S. Dineen, The Schwarz Lemma. The Claredon Press, Oxford, 1989. MR1033739 (91f:46064)

3. V. N. Dubinin, Symmetrization in geometric theory of functions of a complex variable. Uspehi Mat. Nauk 49 (1994), 3-76; English translation in Russian Math. Surveys 49:1 (1994), 1-79. MR.1307130 (96b:30054)

4. F. P. Gardiner and N. Lakic, Quasiconformal Teichmüller Theory. Mathematical Surveys and Monographs, 76. American Mathematical Society, Providence, RI, 2000. MR1730906 (2001d:32016)

5. W. K. Hayman, Subharmonic Functions. Vol. 2. London Mathematical Society Monographs, 20. Academic Press, Inc., London, 1989. MR1049148 (91f:31001)

6. V. Jørgensen, On an inequality for hyperbolic measure and its applications in the theory of functions. Math. Scand. 4 (1956), 113-124. MR.0084584 (18:885a)

7. D. Mejía, Ch. Pommerenke, The analytic point function in the disc. Comput. Methods Funct. Theory 5 (2005), no. 2, 275-229. MR2205415 (2006j:30006)

8. D. Mejía, Ch. Pommerenke, The analytic point function II. Preprint.

9. A. Yu. Solynin, Continuous symmetrization of sets. Zap. Nauchn. Semin. LOMI 185 (1990), 125-139; English translation in J. Soviet Math. 59 (1992), 1214-1221. MR.1097593 (92k:28012)

10. A. Yu. Solynin, Functional inequalities via polarization. Algebra i Analiz 6 (1996), 148-185; English translation in St. Petersburg Math. J. 8:1 (1997), 1015-1038. MR.1458141|(98e:30001a)

11. A. Yu. Solynin and M. Vuorinen, Estimates for the hyperbolic metric of the punctured plane and applications. Israel J. Math. 124 (2001), 29-60. MR.1856503 (2002j:30071)

12. R. Sperb, Maximum Principles and Their Applications. Academic Press, Inc., 1981. MR:0615561 (84a:35033)

Department of Mathematics and Statistics, Texas Tech University, Box 41042, LubBOCK, TEXAS 79409

E-mail address: alex.solynin@ttu.edu 